\title{
Analysis of the Lactose metabolism in E. coli using sum-of-squares decomposition
}

\author{
Ali Ahmadzadeh* ${ }^{*}$ Adam Halasz*, Stephen Prajna ${ }^{\dagger}$ Ali Jadbabaie ${ }^{* \ddagger}$ and Vijay Kumar *
}

\begin{abstract}
We provide a system-theoretic analysis of the mathematical model of lactose induction in E.coli which predicts the level of lactose induction into the cell for specified values of external lactose. Depending on the levels of external lactose and other parameters, the Lac operon is known to have a low steady state in which it is said to be turned off and high steady state where it is said to be turned on. Furthermore, the model has been shown experimentally to exhibit a bi-stable behavior. Using ideas from Lyapunov stability theory and sumof-squares decomposition, we characterize the reachable state space for different sets of initial conditions, calculating estimates of the regions of attraction of the biologically relevant equilibria of this system. The changes in the basins of attraction with changes in model parameters can be used to provide biological insight. Specifically, we explain the crucial role played by a small basal transcription rate in the Lac operon. We show that if the basal rate is below a threshold, the region of attraction of the low steady state grows significantly, indicating that system is trapped in the (off) mode, showing the importance of the basal rate of transcription.
\end{abstract}

\section{INTRODUCTION}

In recent years there has been an explosion of quantitative knowledge on cell-level biochemical processes. Networks of hundreds of reactions have now been mapped. In principle, this allows the construction of mathematical models of regulatory systems determining expressions of genes, and cellular behaviors such as differentiation, responses to environmental signals, and cell-to-cell communication. There are two issues that need to be addressed in order to achieve these goals. One difficulty lies in that while the reactions themselves have been identified, reliable kinetic information is still lacking. Second, if this information eventually becomes available, the complexity represented by the number of reactions and species poses a significant challenge in the construction of traditional models and in the analysis and prediction of the functionality of these networks.

The traditional approach to modelling of genetic networks leads to highly nonlinear systems of differential equations. Simulation-based techniques only provide snapshots of system behavior for specific values of parameters and initial conditions. Thus, such techniques scale poorly as the numbers of species and reactions are increased. With the increasing complexity of the systems under study in systems

* A. Ahmadzadeh, A. Halasz, A. Jadbabaie, and V. Kumar are with the GRASP laboratory, University of Pennsylvania, Philadelphia, PA 19104 We gratefully acknowledge the support of ONR grant YIP-542371, NSF grants ECS-0347285, CNS-0410514, and CCF-0432025, and DARPA grant Biocomp AF F30602-01-2-0563. A. Halasz is supported by an NIH-NLM Individual Biomedical Informatics Fellowship, award 1-F37-LM-008343-1.

$\dagger$ S. Prajna is with the CDS department, Caltech, Pasadena, CA 91125.

$¥$ corresponding author. Email: jadbabai@seas. upenn. edu biology, there is a need for automated software tools to help organize and refine the existing knowledge by creating and analyzing kinetic models of networks of molecules and cells.

In this paper we present an analysis paradigm based on sum of squares (SOS) decomposition that allows the prediction of certain properties of system trajectories without exhaustive simulation. We adopt a kinetic model given by polynomial differential equations. This can be justified because most biological phenomena modelled by reaction rate equations lead to polynomial systems[3]. Our SOS based approach provides a global description of a biological system, helping us gain more insight than by exhaustive simulation or by analysis derived from linearization around equilibria.

We apply the modelling paradigm and the analysis technique to a well-studied example in cellular biology. Our main goal is to develop computational tools that can provide insight into the behavior of such systems without manual calculations. We base our case study on Yildirim and Mackey [9]. They derive a detailed kinetic model of the lac operon and its induction by lactose and show that the system exhibits bistable behavior under certain conditions, but has a single stable steady state otherwise. While simulation results are shown in [9], our focus in this paper is on symbolic analysis. It is typically difficult to obtain the steady states analytically. In order to simplify the model and allow for three dimensional visualization, we develop a three dimensional version of the Lac model studied in [9], by neglecting the "faster dynamics" of two of the states. We then use the SOS technique to characterize the region of attraction of the model equilibria.

Depending on the level of external lactose (the source) and model parameters, the system exhibits three fixed-points, two of which are asymptotically stable (sinks), and one is a saddle. Using SOS, we construct a Lyapunov function for each stable equilibrium and find the largest estimate of the basin of attraction. We then investigate the role of a critical transcription rate and show that if it is below a threshold, the mechanism fails, providing a reasonable explanation of why this rate exists in the first place.

The outline of this paper is as follows. In the next section, we describe the mathematical model for the Lac system taken from [9], and our three dimensional approximation. We illustrate the differences and show how important properties of the original system are preserved with the hybrid systems model. Section IV is devoted to a brief review of the SOS technique and how it can be used for study of basin of attractions. Finally, we provide estimates of the basins of 
attraction of equilibria and pinpoint the role of a critical basal transcription rate.

\section{Modelling}

The state of the biochemical system is represented by a state vector $x=\left[x_{1}, x_{2}, \ldots, x_{n}\right]^{T}$ which is an $n$-dimensional vector of concentrations of all species that are significant descriptors of the behavior of the system. The state equations can be written in the form:

$$
\dot{x}=f(x)
$$

where $f(x)$ is a vector-valued, nonlinear function of the state describing the rate of change of the variables in the system. These rate equations are derived from mass action kinetics, enzymatic reactions and activation functions. Most of these laws are polynomial. Even switches are modelled by smooth functions that are generally ratios of polynomials, such as

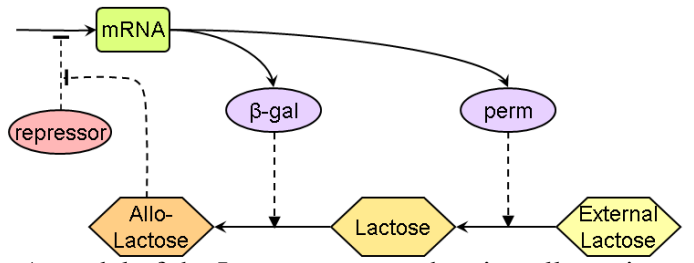

Fig. 1. A model of the Lactose system showing all species, with the arrows denoting processes, with dotted arrows indicating the action of a catalyst. The repressor acts to inhibit the transcription process leading to $m R N A$, while the allolactose inhibits the repressor's action.

Our model of the Lac system is adapted from Yildirim and Mackey [9]. Central to this system is the lac operon which consists of three genes, lacZ, lacY, and $l a c A$ [8]. The enzyme $\beta$-galactosidase, the product of the lac $Z$ gene, cleaves lactose, a necessary first step to metabolize lactose. The $l a c Y$ gene encodes the permease that brings lactose into the cell. In the absence of lactose, Lac repressor binds the lac operator turning down the transcription of its component genes to a low basal level. However, in the presence of lactose, the decomposition of lactose results in the formation of allolactose, which binds to Lac repressor, greatly reducing its binding to DNA. This activates the transcription of the genes to a level that may be as much as 40 -fold higher ${ }^{1}$ the lac than the basal rate. (cf. Figure 1).

We denote the concentrations of the different species in the equations of state (2) as follows. $M$ is the concentration of the mRNA transcribed from both genes, while $B$ and $P$ denote the $\beta$-galactosidase and permease. The external lactose is $L_{e}$, while the intra-cellular lactose is denoted by $L$. Finally, the concentration of allolactose is denoted by $A$. The state of the system is given by:

$$
x=[M, B, A, L, P]^{T},
$$

\footnotetext{
${ }^{1}$ It turns out that the presence of glucose decreases the synthesis of cyclic AMP which in turn affects the ability of catabolite activator protein (CAP) to bind DNA and recruit RNA polymerase to the promoter. Thus, in the presence of glucose, this increase is significant, but much less than 40-fold. We do not consider this feedback loop in our system.
}

while $L_{e}$ is viewed as an input to the system that is controlled outside the cell. The differential equations describing the evolution of the system are:

$$
\begin{aligned}
\frac{d M}{d t} & =\alpha_{M} f_{1}\left(e^{-\mu \tau_{M}} A_{\tau_{M}}\right)+\Gamma_{0}-\tilde{\gamma}_{M} M \\
\frac{d B}{d t} & =\alpha_{B} e^{-\mu \tau_{B}} M_{\tau_{B}}-\tilde{\gamma}_{B} B \\
\frac{d A}{d t} & =\alpha_{A} B g_{1}(L)-\beta_{A} B f_{2}(A)-\tilde{\gamma}_{A} A \\
\frac{d L}{d t} & =\alpha_{L} P h\left(L_{e}\right)-\beta_{L} P g_{2}(L)-\alpha_{A} B g_{1}(L)-\tilde{\gamma}_{L} L \\
\frac{d P}{d t} & =\alpha_{P} e^{-\mu\left(\tau_{P}+\tau_{B}\right)} M_{\tau_{P}+\tau_{B}}-\tilde{\gamma}_{P} P .
\end{aligned}
$$

where $f_{1}(A)$ is a function that captures the effector-repressor dynamics of the transcription enhanced by allolactose,

$$
f_{1}(A)=\frac{1+K_{1} A^{2}}{K+K_{1} A^{2}}
$$

and $f_{2}, g_{1}, g_{2}$ and $h$ are rates of irreversible MichaelisMenten reactions:

$$
\begin{array}{ll}
f_{2}(A)=\frac{A}{K_{A}+A} & g_{1}(L)=\frac{L}{K_{L}+L} \\
g_{2}(L)=\frac{L}{K_{L 1}+L} & h\left(L_{e}\right)=\frac{L_{e}}{K_{L e}+L_{e}}
\end{array}
$$

As in [9], $\alpha$ and $\beta$ denote rate constants for the different processes, the $\tilde{\gamma}$ are coefficients for terms representing decay of species, and $\tau$ denotes delays associated with the finite time required to complete transcription $\left(\tau_{M}\right)$ and translation ( $\tau_{P}$ and $\tau_{B}$ ). For example, if $\tau_{M}$ is the finite time required for the transcription process, $A_{\tau_{M}}$ denotes the concentration of $A \tau_{M}$ prior to the current time. $\Gamma_{0}$ is a fixed basal rate of mRNA transcription. For more details including the significance of these terms, the reader is asked to refer to [9], [1], [2].

So far, our model is identical to the one in [9]. We will now introduce two approximations that will reduce this model to a simpler, system, thus allowing us to study regions of attractions of equilibria.

Our first simplification is to ignore the time delays. The length of the delays associated with transcription and translation is between 0.1 and 2 minutes. Direct simulations of the model [9], [1] suggest a relaxation time of the order of 50 minutes, so that the time delays can be neglected for most dynamical simulations. In the present paper we are primarily interested in the steady state behavior, which is expected to be impacted even less by such small time delays. In particular, the steady states themselves are insensitive to the time delays since the time delayed and prompt variables are equal in this case.

To simplify the model even further and allow for three dimensional visualization, we ignore two of the dynamical equations which are not detrimental to the qualitative behavior of the model. We assume that $B$ and $P$ instantaneously reach their steady state values. This approximation can be interpreted as projecting the problem on the three-dimensional 


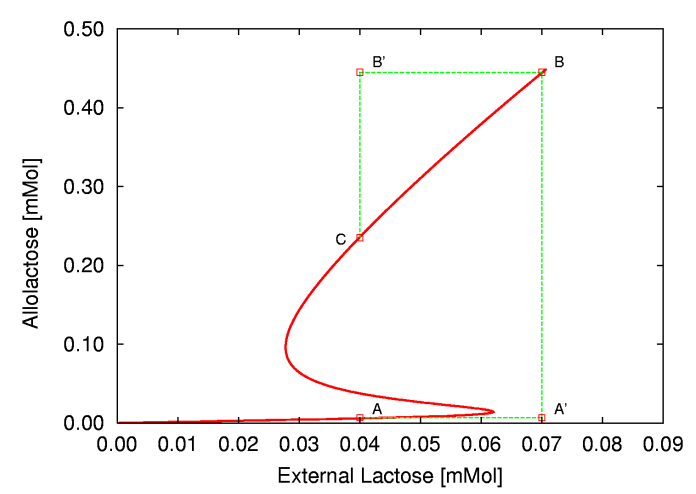

Fig. 2. Steady state values for different external lactos centrations, for the original value of the basal transcription rate. The dotted lines illustrate the approximate trajectory of the system during a change in steady state from $\mathrm{A}$ to $\mathrm{C}$ when driven from a low external lactose concentration to a high concentration, and then back to the same low concentration.

hyperplane defined by

$$
\begin{aligned}
& B=\frac{\alpha_{B} e^{-\mu \tau_{B}}}{\tilde{\gamma}_{B}} M=k_{B} M \\
& P=\frac{\alpha_{P} e^{-\mu\left(\tau_{P}+\tau_{B}\right)}}{\tilde{\gamma}_{P}} M=k_{P} M
\end{aligned}
$$

The respective time constant for $\mathrm{B}$ is not negligible (leading to a relaxation half-life of about $800 \mathrm{~min}$, but only $30 \mathrm{~min}$ if the growth rate is included as indicated in the Appendix). However, in most situations that would occur in nature the $\mathrm{B}$ and $\mathrm{P}$ concentrations are close to their quasi-steady state equilibrium values given below. Our extensive simulations indicate that this approximation does not change the qualitative and quantitative behavior of the model, especially from the stand point of steady state analysis and calculating reachable sets. The simplified model can be written as follows:

$$
\begin{aligned}
\frac{d M}{d t}= & \alpha_{M} f_{1}(A)+\Gamma_{0}-\tilde{\gamma}_{M} M \\
\frac{d A}{d t}= & k_{B} M\left(\alpha_{A} g_{1}(L)-\beta_{A} f_{2}(A)\right)-\tilde{\gamma}_{A} A \\
\frac{d L}{d t}= & k_{P} M\left(\alpha_{L} h\left(L_{e}\right)-\beta_{L} g_{2}(L)\right) \\
& -\alpha_{A} k_{B} M g_{1}(L)-\tilde{\gamma}_{L} L .
\end{aligned}
$$

\section{ANALYSIS OF THE MODEL}

An important result of Yildirim and Mackey [9] was that the system defined by Equations 2 is bi-stable for certain external Lactose concentration values. More recently, this multi-stable characteristic of the Lactose induction mechanism was experimentally verified and quantitatively characterized [4]. This is illustrated by the S-shaped curve in Figure 2 which shows the steady-state values of allolactose for given external Lactose concentrations. We choose $A$ as a measure of the lactose processing activity. Given a putative steadystate value of $A$, the steady-state equations corresponding

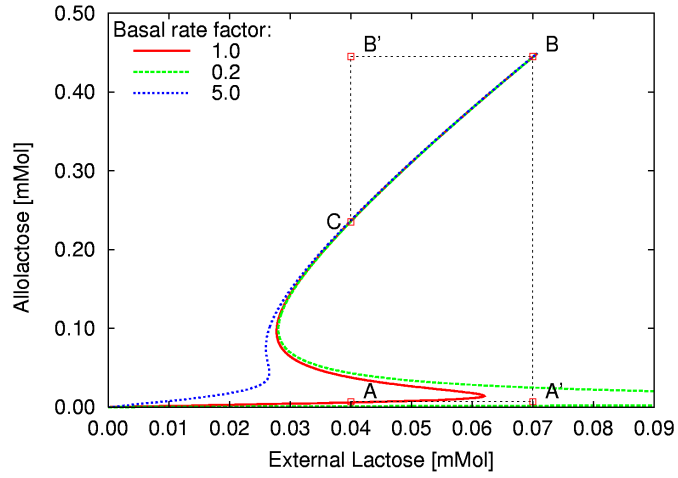

Fig. 3. Steady state values for different external lactose concentrations and basal transcription rates (obtained by changing the factor $b$.

to Equations (2) uniquely determine all four other variables $M, B, L, P$, as well as the corresponding level of external lactose. Similarly, in our reduced model, the equilibrium points of Equations (5-7) allow the steady state values of $M, L$ to be inferred from $A$, which then allow $B$ and $P$ to be determined.

¿From Figure 2 it is easy to see that for a given fixed external lactose concentration we can have either one or three steady states. The negatively-sloped portion of the S-shaped curve in the figure contains unstable states. Thus, in practice, there are at most two steady states for a fixed external lactose concentration, one corresponding to a high metabolic activity level (high $A$ ) and the other a low-activity level (low $A$ ). For very low or very high levels of external Lactose, there is only one steady state for $A$, which is stable. These facts were clearly established in [9]. They were also verified using reachability computations [2].

Suppose the cell is initially in an environment characterized by $L_{e}=0.04 \mathrm{mM}$, hence in the steady state denoted by point A. An increase from to $L_{e}=0.04$ to $0.07 \mathrm{mM}$ will move the system past the "knee" of the curve to the right, after which the steady state will move to the upper branch toward the steady state $B$. Decreasing $L_{e}$ back to $L_{e}=0.04 \mathrm{mM}$ will move the system to the steady state $C$.

A natural question to ask is how sensitive this property is to parameter fluctuations. It turns out that variations up to $20 \%$ produce results that preserve the overall shape of the curve and the bi-stability property. While this result by no means constitutes a mathematical proof, it does provide evidence that this switching property is robust to changes in parameters.

However, there is one parameter that is critical to the bistability property. Small changes in the basal $m R N A$ transcription rate $\Gamma_{0}$ lead to significant changes in the position of the knee of the curve as seen in Figure 3.

We consider different values of the basal transcription rate obtained by scaling the basal transcription rate $\Gamma_{0}^{\prime}=b \Gamma_{0}$. Scaling down this factor $b$ from 1.0 to 0.2 results in the 
knee shifting to the right. Similarly changing $b$ from 1.0 to 5.0 moves the knee to the left. In fact, if the basal rate were further reduced, the level of external Lactose required to effect the jump to the upper branch would increase dramatically and below $b=0.1$ (not shown in the figure) the knee effectively moves to to a value of Lactose that is not realistic. In other words, it is impossible to effect a switch from a low steady state to the high steady state for low values of $b$ (cf. Figure 3). The basal rate of transcription plays the role of a 'pilot light', keeping a minimal amount of permease and $\beta$-galactosidase available even in the total absence of external lactose.

In the following sections we investigate the effects of variations in the basal transcription rate using the SOS programming machinery.

\section{COMPuting Lyapunov Functions AND Regions OF ATTRACTION}

In this section, we will give a brief overview of how a Lyapunov function which proves the stability of $\dot{x}=f(x)$ around its equilibrium can be constructed using sum of squares optimization. We also show how an estimate of the region of attraction for this equilibrium can be obtained.

Without loss of generality, assume that the equilibrium of interest is at the origin (thus $f(0)=0$ ). (If not we can construct the Lyapunov function such that the minimum always happens at the equilibrium). The Lyapunov stability theorem states that if there exists a continuously differentiable function $V: \mathbb{R}^{n} \rightarrow \mathbb{R}$ such that

$$
\begin{aligned}
V(0) & =0, \\
V(x) & >0 \quad \forall x \in D \backslash\{0\}, \\
-\frac{\partial V}{\partial x}(x) f(x) & \geq 0 \quad \forall x \in D,
\end{aligned}
$$

for some neighborhood $D$ of the origin, then the (local) stability of the origin is guaranteed.

When the vector field $f(x)$ is polynomial and the neighborhood $D$ is chosen to be semialgebraic, e.g., of the form $D=\left\{x: g_{D}(x) \geq 0\right\}$ with $g_{D}: \mathbb{R}^{n} \rightarrow \mathbb{R}^{\ell}$ being polynomial, then a polynomial Lyapunov function $V(x)$ can be computed using sum of squares (SOS) programming [7], [6], [5]. The main idea is as follows. First we parameterize $V(x)$ in terms of unknown coefficients, in an affine manner:

$$
V(x)=\sum_{i=1}^{m} c_{i} v_{i}(x)
$$

where the $c_{i}$ 's are the unknown coefficients, and the $v_{i}(x)$ 's are polynomial functions. For example we can choose them to be the monomials in $x$ with degree less than or equal to some degree bound. Once this is done, the various polynomial inequalities that must be satisfied by $V(x)$ are replaced with their corresponding SOS conditions, and the values of $c_{i}$ 's that render $V(x)$ a Lyapunov function are computed by solving the resulting SOS program.

The SOS program which we will use to construct $V(x)$ is stated in the following algorithm [7].
Algorithm 1 (Computing a Lyapunov function): Given is a system $\dot{x}=f(x)$, where $f(x)$ is polynomial and $f(0)=0$. Furthermore, suppose that $D$ is of the form $D=\{x$ : $\left.g_{D}(x) \geq 0\right\}$ with $g_{D}: \mathbb{R}^{n} \rightarrow \mathbb{R}^{\ell}$ being polynomial.

1) Fix a degree bound $2 d$ for $V(x)$, and parameterize $V(x)$ as in (11), where the $v_{i}(x)$ 's are monomials of degree less than or equal to $2 d$.

2) In the same manner, fix a degree bound $2 e$ for a vector of polynomial multipliers $\lambda(x): \mathbb{R}^{n} \rightarrow \mathbb{R}^{\ell}$.

3) Using SOS programming, find the coefficients of $V(x)$ and $\lambda(x)$, as well as some coefficients $\epsilon_{i j}$ such that

(a) $V(x)=0$

(b) $V(x)-\sum_{i=1}^{n} \sum_{j=1}^{d} \epsilon_{i j} x_{i}^{2 j}$ is SOS

(c) $-\frac{\partial V}{\partial x}(x) f(x)-\lambda^{T}(x) g_{D}(x)$ is SOS,

(d) $\lambda_{i}(x)$ is SOS $\forall i=1, \ldots, \ell$,

(e) $\sum_{j=1}^{d} \epsilon_{i j}>0 \quad \forall i=1, \ldots, n$

and $\epsilon_{i j} \geq 0 \forall i, j$.

The polynomial $V(x)$ that we obtain will satisfy (8)-(10), and therefore is a Lyapunov function for the system.

Once the Lyapunov function $V(x)$ is computed, we can use it to estimate a region of attraction of the equilibrium. Here we compute the largest level set of $V(x)$ that is contained in $D$, over which $\frac{\partial V}{\partial x}(x) f(x)$ is non-positive. For this, we again use SOS programming, as described in the following algorithm.

Algorithm 2 (Estimating domain of attraction):

Given are a Lyapunov function $V(x)$ and the set $D=\left\{x: g_{D}(x) \geq 0\right\}$ on which (8)-(10) hold, with $g_{D}: \mathbb{R}^{n} \rightarrow \mathbb{R}^{\ell}$ being polynomial.

1) Parameterize a vector of polynomial multipliers $\lambda$ : $\mathbb{R}^{n} \rightarrow \mathbb{R}^{\ell}$ in terms of some unknown coefficients $c_{i}$ 's.

2) Using SOS programming, find the smallest $\gamma$ such that

$$
\begin{aligned}
& \gamma-V(x)-\lambda^{T}(x) g_{D}(x) \text { is } \operatorname{SOS} \\
& \lambda_{i}(x) \text { is } \operatorname{SOS} \quad \forall i=1, \ldots, \ell .
\end{aligned}
$$

The set $\left\{x \in \mathbb{R}^{n}: V(x) \leq \gamma\right\}$ will be an estimate of the domain of attraction for the equilibrium $x=0$.

\section{COMPUTATION OF BASINS OF ATTRACTION}

The above discussed procedure runs in polynomial time once the degree of the multiplier polynomials as well as the Lyapunov functions are fixed. Our search using SOS programing results in a quadratic Lyapunov functions and sixth order sum of squares polynomials multipliers $(d=1$ and $e=3$ in Algorithm 1) to prove the invariance of the largest level sets of the Lyapunov function inside the set where the derivative is negative. Note that we can not search over the "size" of the region of attraction and the stability certificate simultaneously. Instead, we have to first fix a small region around each equilibrium, use SOS programing 
to find the $V$, by finding the SOS multipliers, and then solve a separate SOS program which grows the level set of $V$. Since a quadratic Lyapunov function was found, there was no reason to look for higher order Lyapunov functions. Of course the better the higher the order of the Lyapunov function, the better the estimate of the region of attraction is going to be.

We repeat this procedure both for the high steady state and the low steady state and estimate the basins of attraction of both the high and low steady states for different values of $\Gamma_{0}$.

Figure 4 shows the largest sub-level set of the two Lyapunov functions corresponding to the low and high steady states, for a basal transcription rate of $\Gamma_{0}^{\prime}=0.2 \times \Gamma_{0}$. The unstable fixed point lies between the two sets, indicating that we can not grow the level sets any further. We now increase the basal transcription rate by a factor of 5 to $\Gamma_{0}$. Figure 5 . depicts the best estimate of the regions of attraction for this scenario. The low region clearly shrinks and the high region grows in some of the directions.

In order to understand the changes in both basins we overlay the two plots on the same scale in Figure 6. The small diamonds indicate the locations of respective steady state points, as well as those of the unstable steady states (same color code). We observe the following two points: (i) The low steady state moves closer to the origin for the reduced basal rate, while the unstable state migrates away from the origin, increasing the distance between the two points. (ii) The size of the estimated region of attraction increases for lower $\Gamma_{0}$. This provides a logical explanation for existence of the basal rate: If its value is smaller than $\Gamma_{0}$, the set of un-inducible states becomes larger. Of course, the true region of attraction is a more complicated surface than an ellipsoid, and the ellipsoid is only an estimate. As a result, there are points in the true region of attraction that are not inside the level set of the largest ellipsoid. For example, in Figure 6 there is a small region included in the blue/cyan surface corresponding to $\Gamma_{0}$ which is not contained in the region described by the red/magenta surface, corresponding to $0.2 \times \Gamma_{0}$. This is due to the conservativeness of the ellipsoidal approximation of the region of attraction. However, we clearly observe the shrinkage of the "uninducible" states as the basal transcription rate is increased.

Finally, Figure 7 depicts the basin of attraction of the high steady state for the same three values of the basal rate $\Gamma_{0}$ discussed above : $\frac{1}{5} \Gamma_{0}, \Gamma_{0}$ and $5 \Gamma_{0}$. Here, the smallest region is the one for $\Gamma_{0}$, and the largest one is for $5 \Gamma_{0}$. This apparently counter-intuitive result can be understood as follows. First, we notice that decreasing the basal rate from $\Gamma_{0}$ will result in migration of the high steady state away from the saddle-point, leading to a higher basin of attraction, mirroring the situation seen for the lower steady state. Secondly, the surface indicating the calculated region of attraction for the basal rate of $5 \Gamma_{0}$ is the largest. This is not surprising since here the high steady state is the only (biologically meaningful) one. However, even in this case the region of attraction is not the whole space, due to the existence of a spurious (mathematically correct but nonphysical) steady state outside the positive octant.

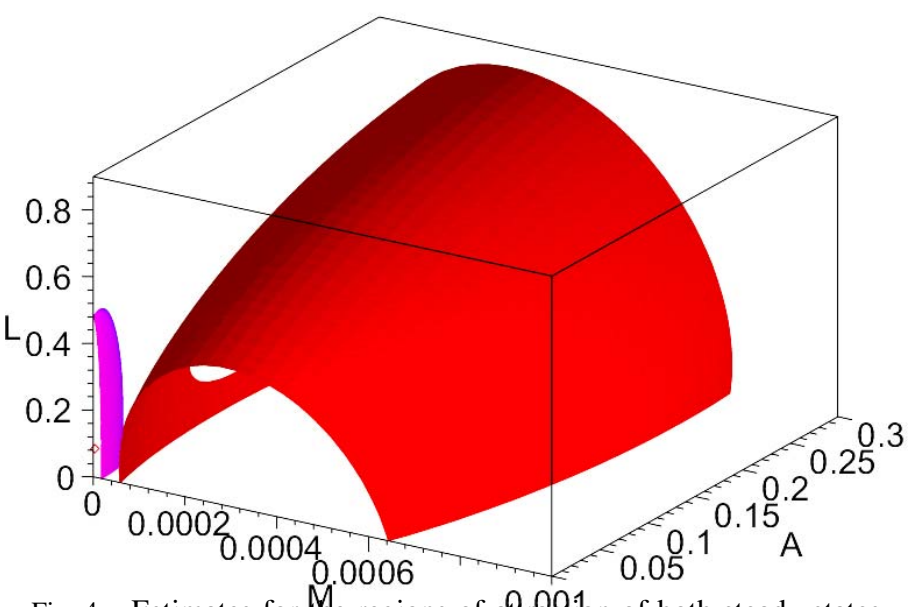

Fig. 4. Estimates for the regions of atraetion of both steady states for a basal rate of $0.2 \times \Gamma_{0}$. The smaller ellipsoid (magenta) is for the lower steady state and the larger (red) is for the higher steady state. The low stable steady state is at $[M, A, L]=\left[.664 \times 10^{-6}, .113 \times\right.$ $\left.10^{-2}, .825 \times 10^{-1}\right]$, and the unstable steady state is between the two regions at $[M, A, L]=\left[.155 \times 10^{-4}, .432 \times 10^{-1}, .176\right]$.

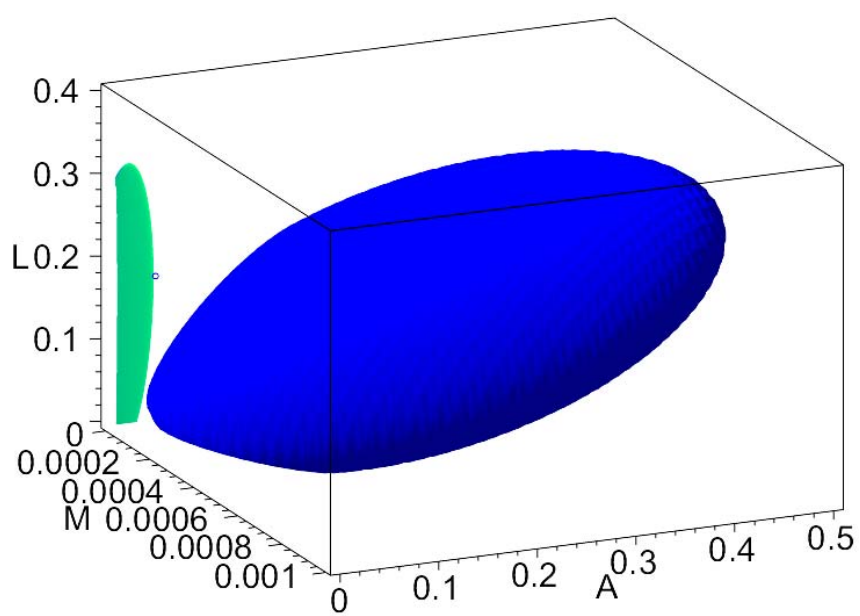

Fig. 5. Estimates for the regions of attraction of both steady states for a basal rate of $\Gamma_{0}$. The smaller ellipsoid (cyan) is for the lower steady state and the larger (blue) is for the higher steady state. The low stable steady state is at $[M, A, L]=\left[.227 \times 10^{-5}, .590 \times\right.$ $\left.10^{-2}, .135\right]$, and the unstable steady state is between the two regions at $[M, A, L]=\left[.133 \times 10^{-4}, .376 \times 10^{-1}, .174\right]$.

In summary, we clearly observe that the basal transcription rate has a significant effect on the size of the region of attraction of both equilibria, especially that of the low steady state which corresponds to un-inducible states.

\section{CONCLUSIONS}

We analyzed the mathematical model of the Lactose metabolism in E.coli using sum of squares programing. The proposed scheme was based on a construction of Lyapunov functions for the stable equilibria of a reduced order model of the system. using sum of squares programing, we characterized regions of attraction estimates for the stable equilibrium 


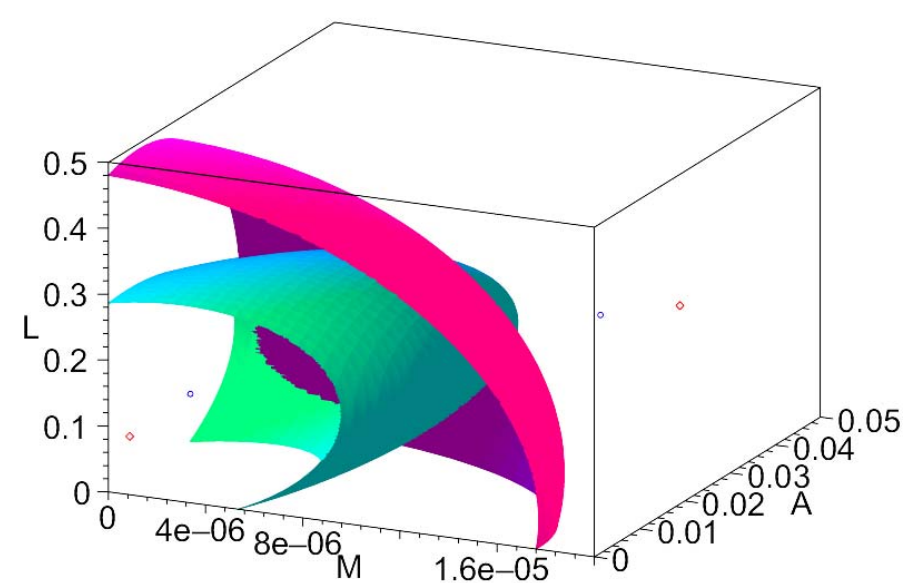

Fig. 6. Estimates of the regions of attraction of the low steady states for basal rates $\Gamma_{0}$ and $0.2 \Gamma_{0}$. The corresponding surfaces are colored in cyan and magenta respectively. For the basal rate of $\Gamma_{0}$, the low steady state and the saddle are depicted as blue diamonds. For basal rate of $0.2 \Gamma_{0}$, the low steady state and the saddle (red circles) migrate away from each other, leading to an overall increase in the region of attraction.

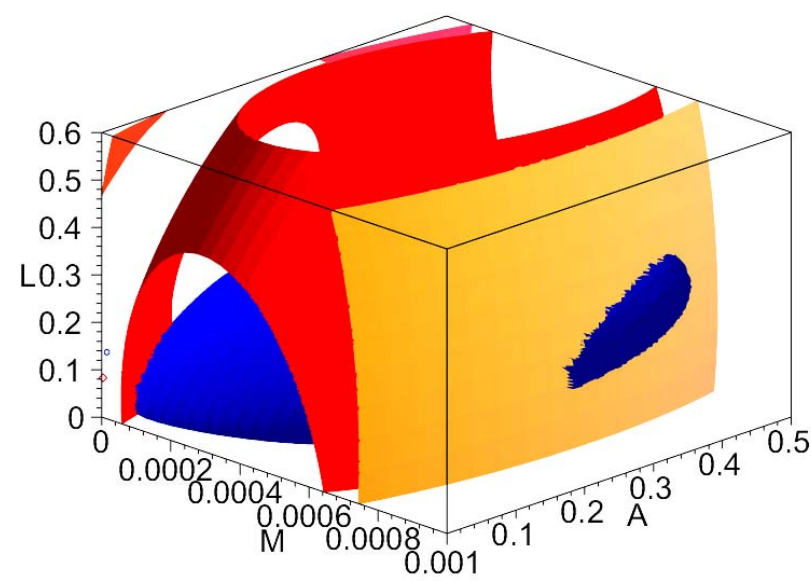

Fig. 7. Estimates for the regions of attraction of the high steady states for the three basal rate values discussed. The smallest ellipsoid corresponds to the middle value of $b=1$ (blue). The middle ellipsoid (red) corresponds to the lowest $b$ value, 0.2 . The largest ellipsoid (yellow) corresponds to $b=5$, when the high steady state is the only meaningful one inside the physically meaningful positive octant.

points. It was shown that the size of the region of attraction critically depends on a basal transcription rate. Decreasing the transcription rate below a certain threshold resulted in significant increase of the region of attraction of the "low" steady state of the system. The impacts of this change on the "high" steady state was also analyzed.

The next step in this direction would be to include the time delays in the analysis and find higher order Lyapunov functions. Another natural direction is to investigate scalability of our approach to systems with larger dimensions. We note that the complexity of the analysis increases polynomially with the degree of the polynomial functions used to describe $f(x)$. On the other hand, the alternative model in [2] allows us to approximate the nonlinear functions using piece-wise affine functions. This approximation requires the analysis of a hybrid system with discrete modes. There is of course an additional growth in the complexity of the analysis because of the number of discrete modes that have to be considered, but the description of the polynomial system in each discrete mode is guaranteed to be of lower order which presumably would simplify the analysis. This interesting trade-off is a promising direction for future investigation.

\section{REFERENCES}

[1] A. Halasz, M. Imielinski, P. Finin, O. Sokolsky, H. Rubin, and V. Kumar. The lactose utilization network of e,coli as a hyrid system: Exploring bistability with reachability analysis. Submitted to FOSBE 2005, Foundations of Systems Biology in Engineering, Santa Barbara, California, August 2005, 2005.

[2] A. Halasz, V. Kumar, M. Imielinski, O. Sokolsky, P. Finin, C. Belta, and H. Rubin. Analysis of lactose metabolism in e.coli using reachability analysis of hybrid systems. Submitted for publication. http: //www.cis. upenn. edu/biocomp/, 2004.

[3] R. Heinrich and S. Schuster. The Regulation of Cellular Systems. Chapman \& Hall, 1996.

[4] E. M. Ozbudak, M. Thattai, H. N. Lim, and B. Shraiman amd A. van Oudenaarden. Multistability in the lactose utilization network of escherichia coli. Nature, 427:737-740, February 2004.

[5] A. Papachristodoulou and S. Prajna. On the construction of lyapunov functions using the sum of squares decomposition. In Proceedings IEEE Conference on Decision and Control, 2002.

[6] Pablo A. Parrilo. Structured Semidefinite Programs and Semialgebraic Geometry Methods in Robustness and Optimization. $\mathrm{PhD}$ thesis, California Institute of Technology, Pasadena, CA, 2000. Available at http: / /www. control.ethz.ch/ parrilo/pubs/index.html.

[7] Stephen Prajna, Antonis Papachristodoulou, and Pablo A. Parrilo. Introducing SOSTOOLS: A general purpose sum of squares programming solver. In Proceedings IEEE Conference on Decision and Control, 2002.

[8] M. Ptashne and A. Gann. Genes and Signals. Cold Spring Harbor Laboratory Press, New York, 2002.

[9] N. Yildirim and M. C. Mackey. Feedback regulation in the lactose operon: A mathematical modeling study and comparison with experimental data. Biophys. J., pages 2841-2851, 2003.

\section{APPENDIX}

The model parameters are the same as those used in [9]. The effective time constants $\tilde{\gamma}$ are obtained by adding the growth rate $\mu$, so that $\tilde{\gamma}_{A}=\gamma_{A}+\mu$ and so on.

\begin{tabular}{|l|c|l|c|}
\hline$\gamma_{M}$ & $0.411 \mathrm{~min}^{-1}$ & $\gamma_{B}$ & $8.33 \times 10^{-4} \mathrm{~min}^{-1}$ \\
$\gamma_{A}$ & $0.52 \mathrm{~min}^{-1}$ & $\Gamma_{0}$ & $7.25 \times 10^{-7} \mathrm{mM} / \mathrm{min}$ \\
$K$ & $7.2 \times 10^{3}$ & $\alpha_{M}$ & $9.97 \times 10^{-4} \mathrm{mM} / \mathrm{min}$ \\
$\tau_{B}$ & $2.0 \mathrm{~min}$ & $\alpha_{A}$ & $1.76 \times 10^{4} \mathrm{~min}^{-1}$ \\
$K_{L 1}$ & $1.81 \mathrm{mM}$ & $\alpha_{B}$ & $1.66 \times 10^{-2} \mathrm{~min}^{-1}$ \\
$K_{A}$ & $1.95 \mathrm{mM}$ & $\beta_{A}$ & $2.15 \times 10^{4} \mathrm{~min}^{-1}$ \\
$\tau_{M}$ & $0.1 \mathrm{~min}$ & $K_{L}$ & $0.97 \mathrm{mM}^{-1}$ \\
$\gamma_{L}$ & $0.0 \mathrm{~min}^{-1}$ & $\gamma_{P}$ & $0.65 \mathrm{~min}^{-1}$ \\
$\alpha_{L}$ & $2.88 \times 10^{3} \mathrm{~min}^{-1}$ & $\alpha_{P}$ & $10.0 \mathrm{~min}^{-1}$ \\
$\tau_{P}$ & $0.83 \mathrm{~min}$ & $\beta_{L}$ & $2.65 \times 10^{3} \mathrm{~min}^{-1}$ \\
$K_{L e}$ & $0.26 \mathrm{mM}$ & $K_{1}$ & $2.52 \times 10^{4} \mathrm{mM}^{-2}$ \\
$\mu$ & $0.0226 \mathrm{~min}^{-1}$ & $k_{B}$ & 0.677 \\
& & $k_{P}$ & 13.94 \\
\hline
\end{tabular}

The steady state values for the three basal rate factors discussed in the text are summarized below.

\begin{tabular}{|l|l|l|l|l|}
\hline State & $\mathrm{b}$ & {$[M]$} & {$[A]$} & {$[L]$} \\
\hline Low & 0.2 & $.664 \cdot 10^{-6}$ & .00113 & .0825 \\
Unst & 0.2 & $.154 \cdot 10^{-4}$ & .0431 & .176 \\
High & 0.2 & $.373 \cdot 10^{-3}$ & .235 & .185 \\
Unst & 1.0 & $.133 \cdot 10^{-4}$ & .0376 & .174 \\
High & 1.0 & $.375 \cdot 10^{-3}$ & .236 & .186 \\
High & 5.0 & $.385 \cdot 10^{-3}$ & .237 & .185 \\
\hline
\end{tabular}

